\title{
A New Approach to the Analysis of Electromagnetic FEM Simulations Results at Electric Field Singularities
}

\author{
Giovanni Betti Beneventi ${ }^{1}$, Massimo DalRe $^{2}$, Luca Vincetti ${ }^{3}$ \\ ${ }^{1}$ At the time of this work was with Federal-Mogul Powertrain Italy, Carpi (MO), Italy. \\ He is now with Tetra Pak Packaging Solutions, Modena, Italy. \\ ${ }^{2}$ Federal-Mogul Powertrain Italy, Carpi (MO), Italy. \\ ${ }^{3}$ University of Modena e Reggio Emilia, ”Enzo Ferrari” Department of Engineering, Modena, Italy. \\ *corresponding author, E-mail: giovanni.bettibeneventi@tetrapak.com
}

\begin{abstract}
A new methodology to the analysis of the results of FiniteElement Modeling (FEM) simulations at electric field singularities is proposed. The method, that can be easily applied in the post-processing phase of the electromagnetic FEM analysis workflow, is based on the weighted averaging of the calculated electric field magnitude within small volumes including the singularity point under investigation. In the paper, the proposed approach is applied to the electrical stress analysis of a high-voltage device modeled by means of a commercial electromagnetic FEM tool. In comparison to the conventional metric of the maximum field evaluation usually adopted for the analysis of electrical stress in insulators, our approach features several advantages: (i) the outcome of the analysis is independent of the numerical grid refinement at the singularity, thus allowing direct comparison of calculated electric field with the material dielectric strength; (ii) the method is robust against slight modifications of the geometrical shape of the singularity; (iii) on the other hand, for a given shape, the analysis outcome responds to significant variations of the singularity size or, in other words, of its sharpness; (iv) in the analysis of highvoltage devices, the approach can be applied for the estimation of the discharge volumes corresponding to different singularity types of different device geometries. In the paper, the new methodology is explained in details and is applied to simple but significant case studies.
\end{abstract}

\section{Introduction}

Singularities at corners or wedges of conductors and dielectrics are manifestations of the vectorial nature of the electromagnetic field. It is a well-known phenomenon which has been subject of intense research for decades [1][3]. The presence of electric field singularities is a problem both from numerical point of view and from insulation design. From numerical point of view, sharp singularities of the electromagnetic field cause difficulties and require ad-hoc solutions hardly ever implemented in the commercial softwares [4]-[8]. This result in a strong dependence of the solution accuracy on the mesh refinement [8]. The local singularity of the electric field also causes problem in the designing of insulators in high power electri-

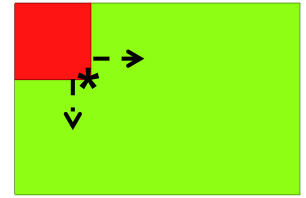

(a) metal-dielectric corner

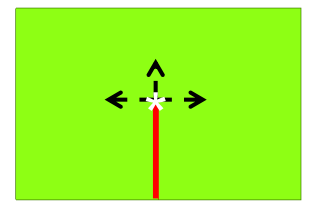

(c) metal tip

$\underline{\text { Legend }}$

$--\rightarrow \hat{\mathbf{n}}$

* singularity

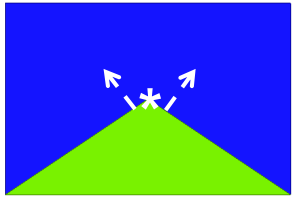

(b) dielectric-dielectric corner

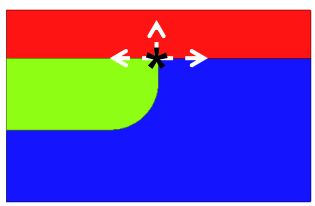

(d) triple point

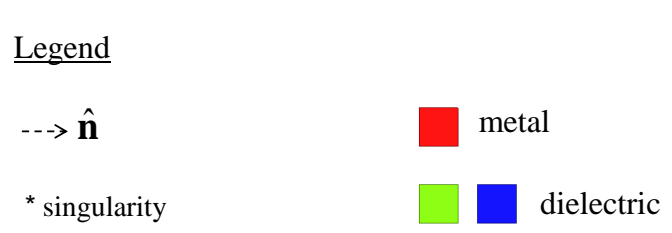

Figure 1: Typical field singularities, indicated by the asterisks $(*)$ : (a) $90^{\circ}$ metal-dielectric corner, (b) dielectricdielectric corner, (c) metal tip, (d) triple point, where three different materials meet at one single point. The unit vector normal to the interface $(\hat{\mathbf{n}})$ is displayed by the dotted arrows. Ambiguous definition of $\hat{\mathbf{n}}$ occurs at the singularity points, where there is more than one arrow pointing to different directions depending on which edge of the corner is taken as reference. For the clarity of illustration, in (a) and (b) the arrows are not drawn exactly at the singularity points, but in their close proximity.

cal and electromagnetic components. The local high electric field can cause surface flash-over and dielectric breakdown [9]. The accurate analysis of regions exposed to high electric field is thus paramount. Typical field singularities frequently encountered in practical electromagnetic problems, e.g. metal-dielectric or dielectric-dielectric corners, metal tips and so-called "triple points", are schematically illustrated in Fig. 1. The main issue with field singularities is that, from a pure analytical point of view, the field at 
singularity diverges. This is of course a mathematical artifact yielding to unphysical results. The artifact is due to the need of representing the material interfaces through idealized sharp corners and tips. The consequence of this simplified representation on the device numerical analysis, e.g. FEM, is that singularities yield field values that do not converge with the increased refinement of the numerical grid applied at the singularity. On the contrary, the field seamlessly increase with the enhancement of the mesh refinement, thus hindering a meaningful assessment of the field values at the singularity.

Nevertheless, singularities are not problematic only from a theoretical point of view. Even though corners and tips in real devices do not have such idealized shapes and the field is never infinite, field peaks occurring at real corners and tips is a well-known physical effect. In many applications, high-field peaks are undesirable since they can cause partial discharge and, ultimately, complete breakdown of the device insulation. Therefore, since numerical computational tools such as FEM are needed to effectively model devices with complex shapes and materials, new approaches allowing to effectively analyze, compare and evaluate the results of FEM simulations concerning the electrical stress at field singularities can be useful.

However, the singularity problem is almost disregarded in the literature concerning the analysis of FEM simulations results of devices and systems where high-voltages and insulation challenges are a concern. In some cases, the calculated electric field peaks at the singularities are reported and directly compared with the material dielectric strength without highlighting that the obtained field values are affected by the chosen numerical mesh size, thus they are not physically meaningful by itself [4].

Alternatively, the most straightforward and commonly used approaches to address this problem are: (1) removing the singularity by modifying the device geometry, e.g. by rounding a sharp corner edge, or (2) limiting the analysis to comparison of electric fields obtained applying the same mesh element size. Then, recently, another method has been suggested which is based on the comparison of the field values at some points belonging to chosen lines in the device geometry [8]. In the latter, the field comparison is done on a coordinate which is implicitly considered from one hand sufficiently far from the singularity point such that the calculated fields are independent of the mesh refinement at the singularity, and, from the other hand sufficiently close to the singularity such that the field is somehow affected by the presence of the singularity itself [8]; hereafter this method will be referred to the Line Method.

In this framework, we aim to propose a new method for the analysis and comparison of fields at singularities that is easily applicable by means of commercial electromagnetics computational tools. We believe that it can provide advantages compared to the above methodologies. Namely, in comparison with (1) and (2), it features: (i) independence of the analysis on the mesh refinement at the singularity, thus allowing direct comparison of the calculated fields with the material dielectric strength $\left(E_{\mathrm{r}}\right)$; (ii) robustness of the analysis against typically unknown details of the singularity shape: (iii) sensitivity of the analysis against significant variation of the singularity sharpness, for a given shape. Then, with respect to the Line Method, our approach accounts for the total field distribution at the singularity rather than limiting the analysis to a specific line. This allows estimating the total volume at the singularity region that might be interested by dielectric breakdown. Indeed, for general combinations of geometries, boundary conditions and material parameters (e.g. dielectric constant), similar fields might be sustained on different volumes. Estimation of the volume possibly interested by breakdown and then discharge is of great interest when the reliability of different device designs is under investigation.

The paper is organized as follows. Section 2 describes the Device Under Test (DUT) that we adopt as a benchmark of the new proposed modeling approach. Section 3 discusses more in-depth the limitations of the usual methodologies reported above. Then, in section 4, the method is explained in details in order to show its application to simple but meaningful test cases. Our methodology is benchmarked with respect to mesh-independence, robustness, and sensitivity. Next, a discussion of the applicability of the method in the industrial practice is provided in section 5. Finally, section 6 draws the conclusions of this work.

\section{The device under test}

The Device Under Test (DUT) is a high-voltage coaxial insulator composed by several dielectric layers. It has cylindrical symmetry with respect to the longitudinal rotational axis $(z)$, thus we can model only half of the device crosssection to be solved in cylindrical coordinates. The considered singularity point is a $30^{\circ}$-angle triple-point located at the interface between a metal and two dielectrics, see Figure 2. The metal, accounted for in the model by the applied voltage boundary condition at radial coordinate $r_{\mathrm{V}}>0$, represents the central electrode of the coaxial structure. Therefore, the electric field magnitude $(E)$ is higher in correspondence to the central electrode then it decreases approximately as $1 / r$, being $r$ the radial coordinate. Therefore, the electric stress nearby the central electrode must be carefully evaluated. Consider also that the "dielectric-1" has a dielectric constant which is about 5 times lower the one of the "dielectric-2" material, hence it undergoes higher fields. Moreover, the triple-point singularity is the most critical region of dielectric-1, being characterized by the maximum electric field in the investigated domain $\left(E_{\mathrm{MAX}}\right)$ due to the sharpness of its shape.

Given the DUT symmetry, the electromagnetic problem is virtually solved in 3-D by means of expressing the model in cylindrical coordinates. For this reason, in the following we refer either to "surfaces" when describing the geometry and field distribution on the cross-section or to "volumes" referring to the integration of surface quantities by means of the rotation on the $z$-axis. Then, we define two subdomains in the DUT section, the "mesh surface" and the "control sur- 


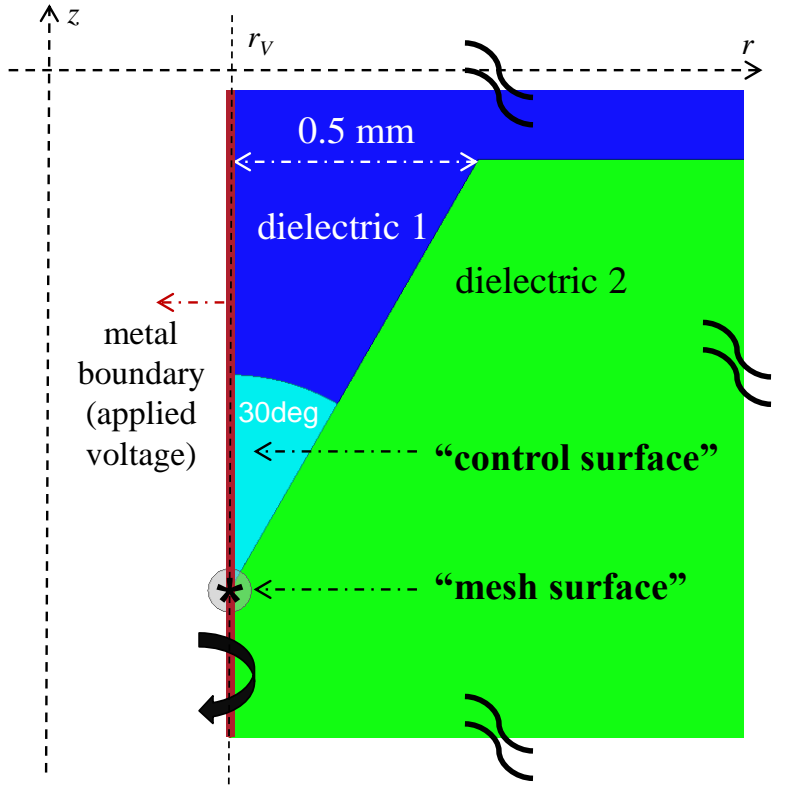

Figure 2: Part of the DUT containing the singularity to which the new approach is applied. Given the axialsymmetry with respect to the longitudinal $z$ axis, the drawing is limited to only half of the cross section. The lefthand side metal boundary, to which a voltage is applied, represents the coaxial central electrode, and is located at a radial coordinate $r=r_{\mathrm{V}}>0$, while a zero-voltage condition is applied to the ground shield at a radial coordinate $r_{0}>>r_{V}$ (not shown in the figure). The singularity under investigation is the triple-point indicated with the asterisk (*). The triple-point singularity consists in a sharp edge of $30^{\circ}$ angle, which end-tip is located on the left interface and is common to both the metal and the two dielectrics. The triangular-like aperture has maximum radial dimension of about $0.5 \mathrm{~mm}$, while the maximum DUT dimension features several tens of centimeters. The circular sector which includes the singularity, and that is centered at the singularity point $(*)$, is the so-called "control surface" to which corresponds the "control volume" obtained by the rotation of the control surface on the $z$-axis. The control surface, of variable area, is where the magnitude of the electric field is averaged in a post-processing step. In the FEM physical model, the control surface is part of "dielectric 1". Besides, the circle drawn in transparency and centered on the singularity point, represents the "mesh surface". Like the control surface, the mesh surface domain is not modeled in the electrostatic solver and serves only in the simulation pre-processing phase to set the numerical grid maximum element length ( $\left.L_{\mathrm{MAX}}\right)$ nearby the singularity.

face", see Figure 2. In this example, the mesh surface and the control surface are a circle and a circular sector, respectively, centered at the singularity point. It must be pointed out that these subdomains do not affect the solution of the electrostatic model, both are so-called non-model domains used only for mesh definition and post-processing of re- sults, respectively. Indeed, the "mesh surface" is where the $L_{\mathrm{MAX}}$ parameter is applied to control then numerical grid, while the "control surface", generating the "control volume" by rotation, is the part of the cross-section to which we apply the field averaging procedure, as described in the following section.

To conclude, for the sake of simplicity, the problem is solved by applying an electrostatic model by means of a commercial FEM tool, i.e. Ansys Maxwell release 17.1. Nevertheless, the proposed method can be directly applied to a general electromagnetic model. Furthermore, it could be applied to all physical models featuring field singularities, like, as an example, mechanical stress analysis.

\section{Conventional approaches}

\subsection{Modification of the device geometry}

One of the classical workaround to cope with $90^{\circ}$ metaldielectric corner like the one displayed in Figure 1(a) is the application of the so-called fillet operation. This procedure is reported as best practice in most FEM simulators tutorials. The fillet operation consists in transforming the $90^{\circ}$ corner into an arc prior of performing the FEM analysis. However, this method does not actually remove the problem for the following reasons:

- When the corner is rounded other two corners, though not of the $90^{\circ}$ type, are generated at the arc insertion point, thus, to be rigorous, in this way the number geometrical singularities increase from one to two;

- In many numerical packages, the curvilinear elements, which are specifically suited to describe round shapes, are not available. Thus, even though in the geometry modeler the $90^{\circ}$ corner is transformed into an arc, the numerical mesh describing the curve is drawn by means of a series of lines, which edges are connected by corners. Therefore, the fillet operation cannot ensure the removal of the singularity. This issue is usually neglected by the users since the investigated range of mesh refinement at the arc corners is typically restricted. Furthermore, in certain simulation tools, the number of polygons used to describe the arc increases for enhanced mesh refinement, such that the arc discretization gets better for finer meshes; if the better curve discretization is dominant over enhanced refinement, the rate of the field increase with the finer mesh decreases and may be difficult to detect;

- When dealing with interfaces or geometrical details, it cannot be always trivial to effectively apply rounding operations, either because the real curvature of the shape is unknown, or because parts of the geometry should really be modeled as sharp corners to properly conserve the aspect ratio with respect to other device dimensions. Also, as reasonable, software pack- 
ages usually set limitations on object aspect ratio to limit the computational burden.

\subsection{Comparison of maximum field}

Another easy approach is to compare the ratio of the $E_{\mathrm{MAX}}$ at the singularities to perform comparative analysis among different singularities present in a same device, or among different singularities of different devices. Obviously, by limiting the analysis to the relative values of the field at singularities it is not possible to directly compare the calculated field with the material $E_{r}$, as instead it would be

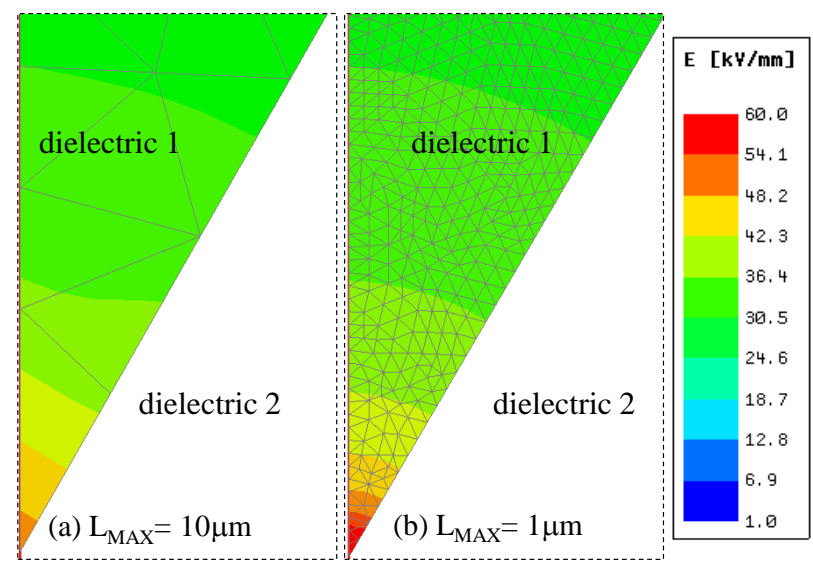

Figure 3: Electric field $(E)$ at the "dielectric 1" side of the mesh surface region of the DUT shown in Figure 2; superimposed is the numerical mesh: (a) mesh with $L_{\mathrm{MAX}}=10$ $\mu \mathrm{m}$, (b) mesh with $L_{\mathrm{MAX}}=1 \mu \mathrm{m}$. The colormap legend ranges from $1 \mathrm{kV} / \mathrm{mm}$ to $60 \mathrm{kV} / \mathrm{mm}$.

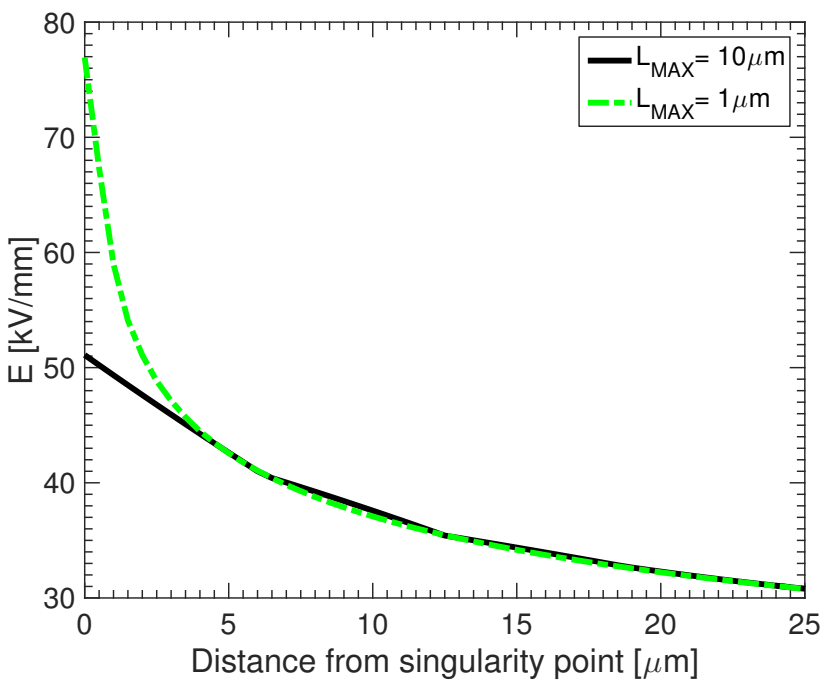

Figure 4: Electric field profiles along the "dielectric 1""dielectric 2" interface for $L_{\mathrm{MAX}}=10 \mu \mathrm{m}$ (continuous line) and $L_{\mathrm{MAX}}=1 \mu \mathrm{m}$ (dot-dashed line). $E_{\mathrm{MAX}}$ is located at the singularity point, then $E$ decreases with increased distance from singularity.

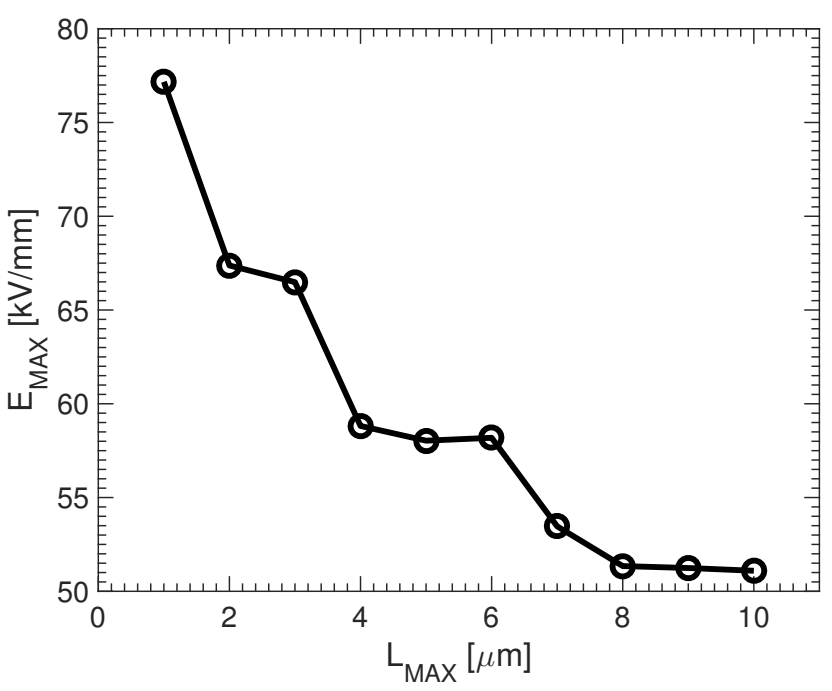

Figure 5: $E_{\mathrm{MAX}}$ vs. $L_{\mathrm{MAX}}$.

needful to assess discharge or breakdown probability. To be fair, this method must be applied by using the same mesh refinement for all the singularities under test. Indeed, to appreciate the dependence of $E_{\mathrm{MAX}}$ against mesh refinement consider Figure 3, which displays the magnitude of the electrostatic field $(E)$ in the DUT region of interest. Figure 3(a) shows the field distribution calculated by using a mesh maximum element length $L_{\mathrm{MAX}}=10 \mu \mathrm{m}$, while Figure 3(b) shows the field distribution for the same geometry, material and boundary conditions, but using a more refined mesh $\left(L_{\mathrm{MAX}}=1 \mu \mathrm{m}\right)$. It is clear how to lower $L_{\mathrm{MAX}}$ correspond higher $E$. To clarify better, Figure 4 shows the electric field along the interface between the two dielectrics varying $L_{\mathrm{MAX}}$ : $E_{\mathrm{MAX}}$ is always located at the singularity point, than $E$ decreases for higher distance from the singularity point. Therefore, from both Figure 3 and Figure 4 it is clear how to an increased refinement corresponds a higher $E_{\mathrm{MAX}}$. This behavior is further investigated in Figure 5, which displays the calculated $E_{\mathrm{MAX}}$ as a function of $L_{\mathrm{MAX}}$. To a decrease of a factor of 10 of $L_{\mathrm{MAX}}$ corresponds an increase of $E_{\mathrm{MAX}}$ of about the $25 \%$.

\subsection{The "Line Method"}

A more recent approach to the singularity problem has been recently reported in literature [8]. The idea behind the Line Method is to analyze the electric field variations with the numerical mesh on a given line arising from the singularity point and extending into the surrounding insulator. Then, the record of the field value characterizing the singularity point is made sufficiently far from the singularity such that, at the probing point, the calculated field does not depend on the mesh refinement. This is a simple and effective approach since the field nearby the singularity is dominated by the singularity itself, which can be conceived as a point charge, therefore the field distribution is approximately radial, i.e. it does not depend on the angular coordinate. On the other hand it might be useful to calculate the total 
volume where the field overcomes the material dielectric strength, hence where partial discharge can occur. In general, the bigger the volume interested by partial discharge, the lower the durability of a device for a given working condition. In general, there can be cases where the combination of different geometries, boundary conditions and material parameters (e.g. the dielectric constants) could yield close electric field values at the singularity, but sustained within different volume sizes. Therefore, we believe that it is useful to propose a general method which, by investigating the field at the singularity, intrinsically estimates the total volume interested by the discharge process.

\section{A new approach}

\subsection{Weighted field-averaging within a control volume}

We analyze the electric stress at the singularity by calculating the average value of the electric field magnitude on a small volume including the singularity point, namely the control volume $\Omega$, as

$$
E_{\mathrm{AVG}}=\frac{\int_{\Omega} E d \Omega}{\int_{\Omega} d \Omega} .
$$

The aim is to find out an $E_{\mathrm{AVG}}$ which is both (i) independent of the mesh refinement, and, (ii) at the same time, representative of the electric stress at the singularity. Obviously, requirement (i) holds as long as $\Omega$, or better, its equivalent cubic side $S_{\text {eq }}=\Omega^{1 / 3}$ is sufficiently bigger than $L_{\text {MAX }}$ and, conversely, requirement (ii) holds as long as $\Omega$ is sufficiently small to be sensitive on the field concentration at the singularity. In other words, for too small $\Omega$ approaching the single element volume, $E_{\mathrm{AVG}}$ tends to $E_{\mathrm{MAX}}$, thus depends on $L_{\mathrm{MAX}}$; on the other hand, for too big $\Omega$, we expect $E_{\mathrm{MAX}}$ at singularity to be hidden by the whole field distribution, hence we expect $E_{\mathrm{AVG}}$ to be basically uncorrelated with the $E_{\mathrm{MAX}}$, thus not providing relevant information about the singularity. Therefore, as obvious, the $E_{\mathrm{AVG}}$ behavior is strictly related to the definition of $\Omega$, which should be properly set to provide the required characteristics. A quantitative analysis of the suitable relative dimensions of $S_{\text {eq }}$ and $L_{\mathrm{MAX}}$ is illustrated next. For the time being, let us assume to have properly defined $\Omega$ to fulfill the above partially conflicting requirements on $E_{\mathrm{AVG}}$.

\subsection{The method in steps and analysis interpretation}

In the following, the operative steps and the best practice to apply in order to implement the proposed method in a commercial FEM computational tool are reported in details. Also, we discuss how the results of the analysis can be interpreted.

1. Define a surface containing the singularity where to apply the controlled mesh refinement through the $L_{\text {MAX }}$ parameters, i.e. the "mesh surface", as represented in Figure 2. The default $L_{\text {MAX }}$ must be chosen as a reasonable baseline in order to limit the computational burden and to fulfill the software maximum aspect ratio specification. To address the DUT, which has maximum dimension in the order of tens of centimeters, an $L_{\mathrm{MAX}}=10 \mu \mathrm{m}$ is reasonable. The mesh surface radius must be small compared to the device dimensions, but not too small, since it must contains a significant number of triangles even when refinement is relatively coarse, e.g. a reasonable choice is take a surface which radius or equivalent side is about 3 times the $L_{\mathrm{MAX}}$;

2. Define another, bigger, surface which contains both the singularity and the mesh surface, i.e. the "control surface" to which corresponds the control volume $\Omega$, as indicated in Figure 2. In the following we provide an analysis of the proper dimensioning of $\Omega$. For the time being, let us assume that a reasonable baseline is tho choose $\Omega$ such that $S_{\text {eq }}$ is always higher than 5 times the reference $L_{\mathrm{MAX}}$;

3. Perform the simulations varying $\Omega$ on a significant range. Obviously, given the discussed restrictions on $L_{\mathrm{MAX}}$ and the relation that must hold between $S_{\text {eq }}$ and $L_{\mathrm{MAX}}, \Omega$ cannot be arbitrarily varied;

4. Reduce $L_{\mathrm{MAX}}$, e.g. of a factor of 2,5 or 10 (i.e. in this example, $L_{\text {MAX }}$ could be reduced down to 1 $\mu \mathrm{m})$, and check the $E_{\mathrm{AVG}}$ dependence on $L_{\mathrm{MAX}}$.

At this point, the analysis is performed, and two are the possible interpretations:

1. $E_{\mathrm{AVG}}$ equals $E_{\mathrm{r}}$ for a given $\Omega$, and, at the same time, it does not depend on $L_{\mathrm{MAX}}$. Therefore, it is possible to define $\Omega_{\mathrm{C}}$ as the critical $\Omega$ for which $E_{\mathrm{AVG}}$ equals $E_{\mathrm{r}}$. If so, discharge is likely to occur in the whole $\Omega_{\mathrm{C}}$, with $E_{\mathrm{MAX}}$ that can only be higher than $E_{\mathrm{r}}$. Similarly, an equivalent critical cubic side can be defined as $S_{\text {eq, C }}=\left.S_{\text {eq }}\right|_{\Omega=\Omega_{\mathrm{C}}}$. Therefore, the following is the analysis outcome:

- The discharge probability within $\Omega_{\mathrm{C}}$ is high;

- $\Omega_{\mathrm{C}}$ is a general metric of the electrical stress at the singularity. It can be used to compare different singularities in the same device, or different singularities in different devices without suffering of the mesh-dependence issue. Also, it fully accounts for the whole field distribution around the singularity, it does not restrict the analysis to a given chosen direction;

2. The calculated $E_{\mathrm{AVG}}$ is always lower than $E_{r}$ in the investigated $\Omega$ range, or, alternatively, $E_{\mathrm{AVG}}$ equals $E_{r}$ only in a range in which $E_{\mathrm{AVG}}$ depends on $L_{\mathrm{MAX}}$. Interpretation:

- If there is an $\Omega$ such that $E_{\mathrm{AVG}}$ equals $E_{\mathrm{r}}$, it holds only within an $\Omega$ range where the results are dependent of the mesh refinement. This fact might ultimately be considered an artifact due 
to the idealized singularity shape, suggesting that, in the real device, discharge probability might be low. At least, it logically will be lower than the discharge probability of a test case contemplated in the above option 1) .

To conclude, for the case 1 ), the $E_{\mathrm{AVG}}$ analysis provides the following important information: within a known volume surrounding the singularity point, the critical volume $\Omega_{\mathrm{C}}$ the electric field equals the material dielectric strength, with the field at the singularity that can only be higher than the $E_{r}$. The analysis based on $\Omega_{\mathrm{C}}$ evaluation is a general robust metric, that does not depend on the employed mesh, and that can be used to compare critical singularity points among different device geometries, for different singularity shapes, and for general field distribution at the singularity. To finish on the illustration of the operative procedure, it is worth noticing that some tools for electromagnetic FEM simulations, like Ansys Maxwell, adopt an adaptive mesh algorithm. This means that, in order to avoid the user to take the care of defining a detailed mesh strategy, the simulator automatically refines the initial user-defined mesh in subsequent iterations up to convergence. The simulation is therefore typically performed by means of several iterations, and after each iteration the software refines the mesh where it is needful. As a consequence, the mesh parameters set by the user before launching the simulation are usually altered, specifically the mesh is refined, thus the user-defined $L_{\text {MAX }}$ can be bigger than the $L_{\mathrm{MAX}}$ actually employed at the last iteration. An effective workaround to ensure global model convergence, and to have the control on $L_{\mathrm{MAX}}$ is adopting the following scheme:

1. Simulate the device up to convergence without defining a mesh surface nearby the singularity under test and save the numerical grid;

2. Load the previously saved numerical grid and define the mesh surface including the singularity (i.e., define $L_{\mathrm{MAX}}$ ), such that the mesh at the singularity is further refined;

3. Re-simulate the device with the new numerical grid by forcing the simulator to perform one single simulation, such that the adaptive mesh refinement is switched-off and $L_{\text {MAX }}$ remains unaltered.

The simulations shown next have been performed by implementing the above methodology in Ansys Maxwell.

\subsection{Independence upon mesh refinement}

Figure 6 and Figure 7 show the results of the application of the new approach to our DUT. Figure 6 displays $E_{\mathrm{AVG}}$ vs. $\Omega$ varying $L_{\mathrm{MAX}}$, while, Figure 7 shows $E_{\mathrm{AVG}}$ as a function of $S_{\text {eq }}$. It is evident how $E_{\mathrm{AVG}}$ is practically independent of $L_{\mathrm{MAX}}$ in the investigated $\Omega$, or $S_{\text {eq }}$, -range, on a wide $L_{\mathrm{MAx}}$ interval. Then, in the figure, the horizontal line indicates the "Dielectric 1" strength $E_{r}$, which, in this example, equals $24 \mathrm{kV} / \mathrm{mm}$. The vertical line instead,

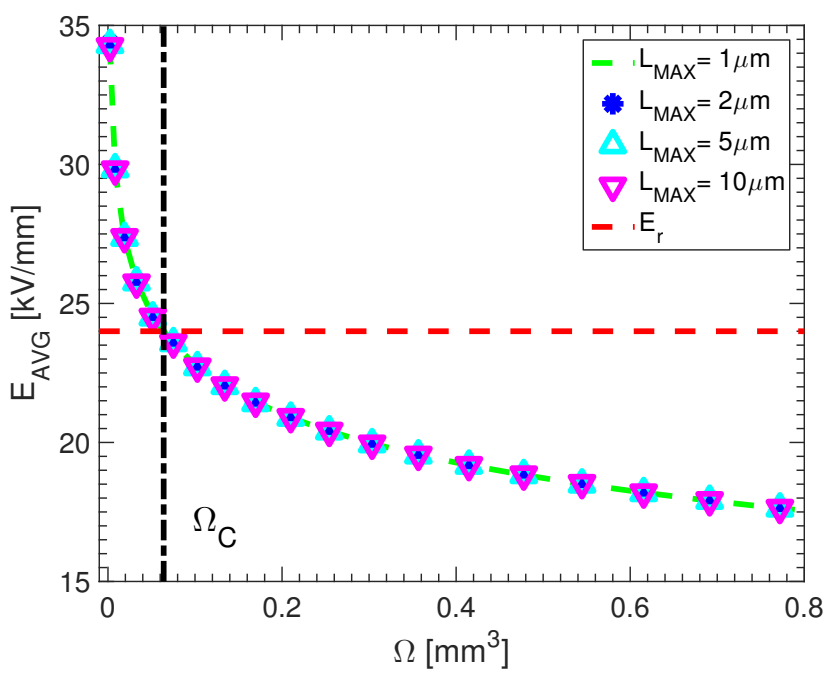

Figure 6: Average electric field $E_{\mathrm{AVG}}$ as a function of the control volume $\Omega$. The critical control volume $\Omega_{\mathrm{C}}$ is the $\Omega$ where $E_{\mathrm{AVG}}=E_{r}$, where $E_{r}$ is the material dielectric strength.

indicates for which value of $\Omega\left(S_{\text {eq }}\right)$, namely $\Omega_{\mathrm{C}}\left(S_{\text {eq, C }}\right)$, the calculated $E_{\mathrm{AVG}}$ equals $E_{r}$, see Figure 6 (Figure 7 ), respectively. Therefore, the analysis results fall in the first interpretation case illustrated in section 4.2, suggesting high discharge probability within $\Omega_{\mathrm{C}}$, with dielectric breakdown likely to occur in "dielectric 1 ".

As shown, in this case, the $E_{\mathrm{AVG}}$-metric is independent of the mesh refinement at the singularity. This property enables fair comparison of the simulated absolute field values against the known $E_{r}$, differently from the $E_{\mathrm{MAX}}$ analysis, which allows only relative field evaluations and that cannot address comparison with $E_{\mathrm{r}}$. The $E_{\mathrm{AVG}}$ metric can thus be used to compare different singularities belonging to different parts of the device or belonging to different devices by assessing the respective $\Omega_{\mathrm{C}}$. The higher the $\Omega_{\mathrm{C}}$, the higher the electrical stress in the dielectric material.

Concerning the criterion for the choice of $\Omega$, we have shown that in this typical example it is possible to determine an $\Omega_{\mathrm{C}}$ belonging to an $\Omega$-range in which $E_{\mathrm{AVG}}$ does not dependent of the employed $L_{\mathrm{MAX}}$. Therefore the outcome of the study is well established, i.e. high probability of dielectric breakdown within $\Omega_{C}$ and no further investigation on other $\Omega$-range would be needed. Nevertheless, it is interesting to investigate better the $L_{\mathrm{MAX}}-\Omega$ behavior for small $\Omega$ to understand for which $S_{\text {eq }}$ values the $E_{\mathrm{AVG}}$-metric suffers from dependence on the numerical mesh. In Figure 8 the $E_{\mathrm{AVG}}$ vs. $S_{\text {eq }}$ behavior is investigated for very low $S_{\text {eq }}$ values, being the minimum $S_{\text {eq }}$ equals to about 1.5 times the maximum chosen $L_{\mathrm{MAX}}=10 \mu \mathrm{m}$. As expected, for low $S_{\text {eq }}$, i.e. $S_{\text {eq }}<50 \mu \mathrm{m}$ corresponding to about 5 times the reference $L_{\mathrm{MAX}}$, the $E_{\mathrm{AVG}}$ curves are no more superimposed, and the $E_{\mathrm{AVG}}$ increases for decreasing $L_{\mathrm{MAX}}$. These results confirms the rule of thumb on the choice of $\Omega$, or better of $S_{\text {eq }}$, which has been suggested in section 4.2. 


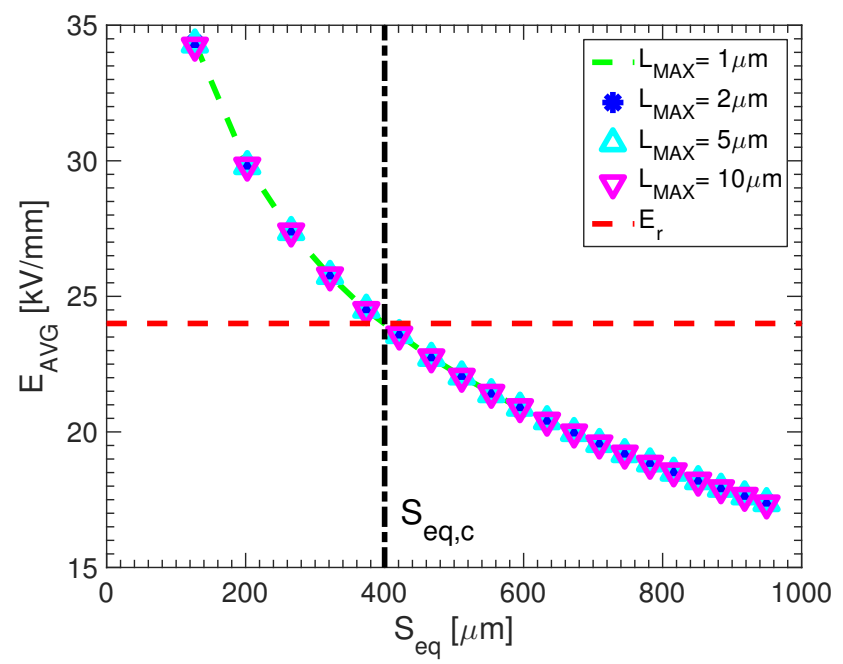

Figure 7: Average electric field as a function of the control volume equivalent cubic side $S_{\text {eq }}=\Omega^{1 / 3}$. The critical equivalent cubic side $S_{\mathrm{eq}, \mathrm{C}}$ is the $S_{\mathrm{eq}}$ where $E_{\mathrm{AVG}}=E_{r}$.

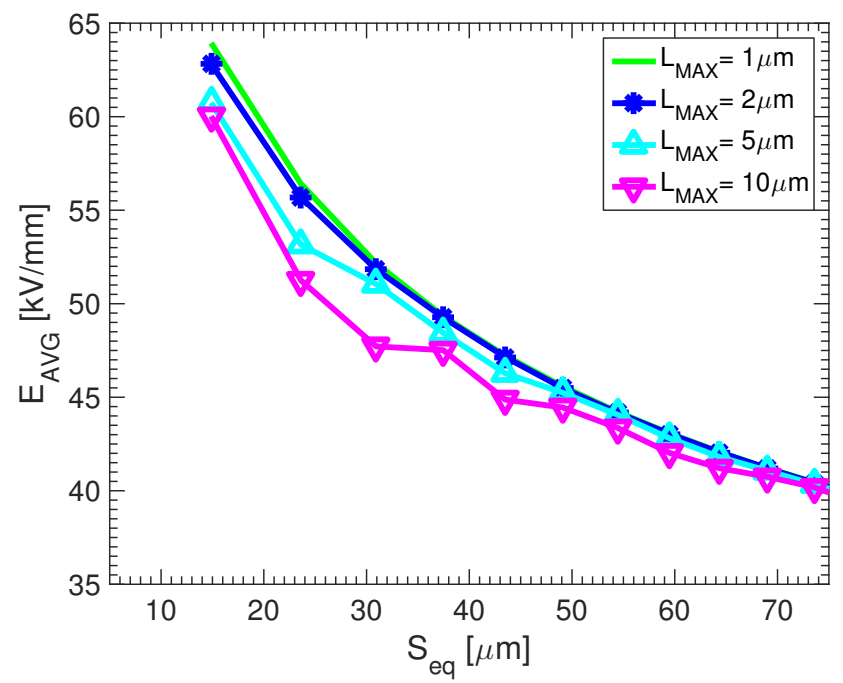

Figure 8: $E_{\mathrm{AVG}}$ as a function of $S_{\mathrm{eq}}$ varying $L_{\mathrm{MAX}}$, for small $S_{\text {eq }}$. In this example. for $S_{\text {eq }}<50 \mu \mathrm{m}$, corresponding to about 5 times the reference $L_{\mathrm{MAX}}$, the $E_{\mathrm{AVG}}$ metric suffers from mesh-dependence.

\subsection{Robustness}

Owing to process variability, the exact profile of the singularity at the interfaces can differ from the as-designed shape. In the following, the proposed method is tested to investigate its robustness with respect to slight variations of the geometric shape at the singularity point.

To perform this test, let us consider two variations applied to the chamfer-like triple-point singularity investigated so far: a truncated-chamfer and fin-chamfer. Figure 9 (a1), (b1), and (c1) shows the profile of the original chamfer, of the truncated-chamfer, and of the fin-like chamfer, respectively. It is worth noticing that the new variations feature a different singularity nature: while the chamfer is a triple-
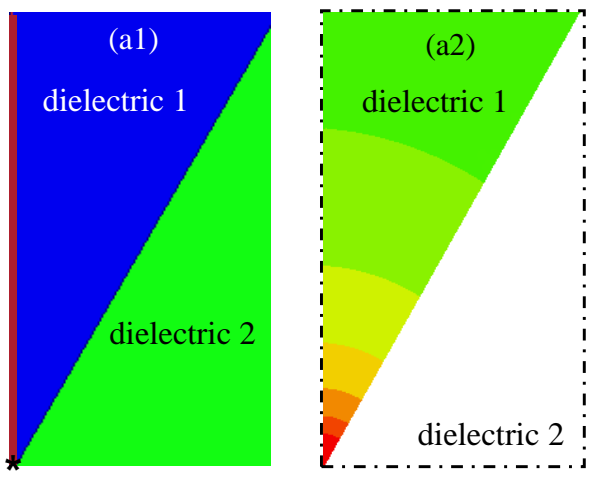

(a) Chamfer
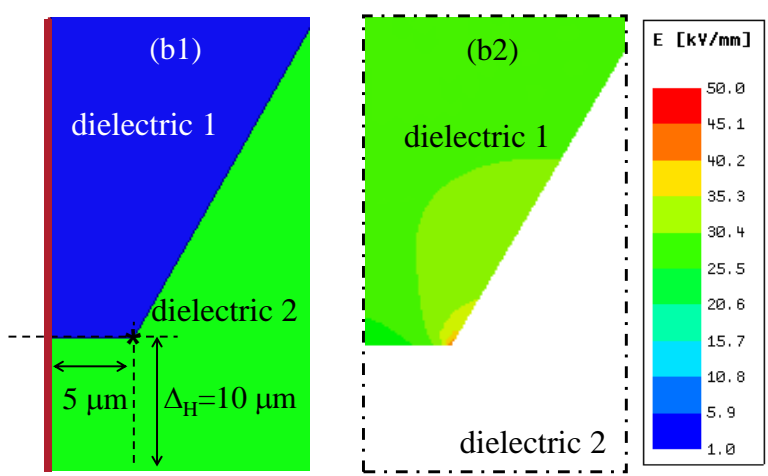

(b) Truncated chamfer
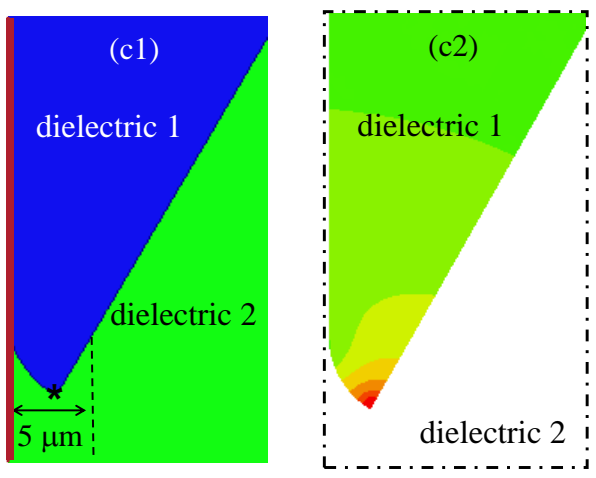

(c) Fin chamfer

Figure 9: (a) Chamfer, (b) Truncated-chamfer, and (c) Fin-chamfer singularity shapes; the chamfer is a triplepoint singularity, while the truncated-chamfer and the finchamfer are dielectric-dielectric corners. Figs.(a1), (b1), and (c1) show the geometrical shapes, where the asterisks (*) indicate, as usual, the singularity points. As shown in Figure (b1), the truncated-chamfer shape feature a "truncation height" $\Delta_{\mathrm{H}}=10 \mu \mathrm{m}$. In the application of the proposed method, the control volume is centered at the respective $(*)$ for each shape. Figs. (a2), (b2), and (c2) display $E$ in "dielectric 1". The colormap legend ranges from $1 \mathrm{kV} / \mathrm{mm}$ to $50 \mathrm{kV} / \mathrm{mm}$. 


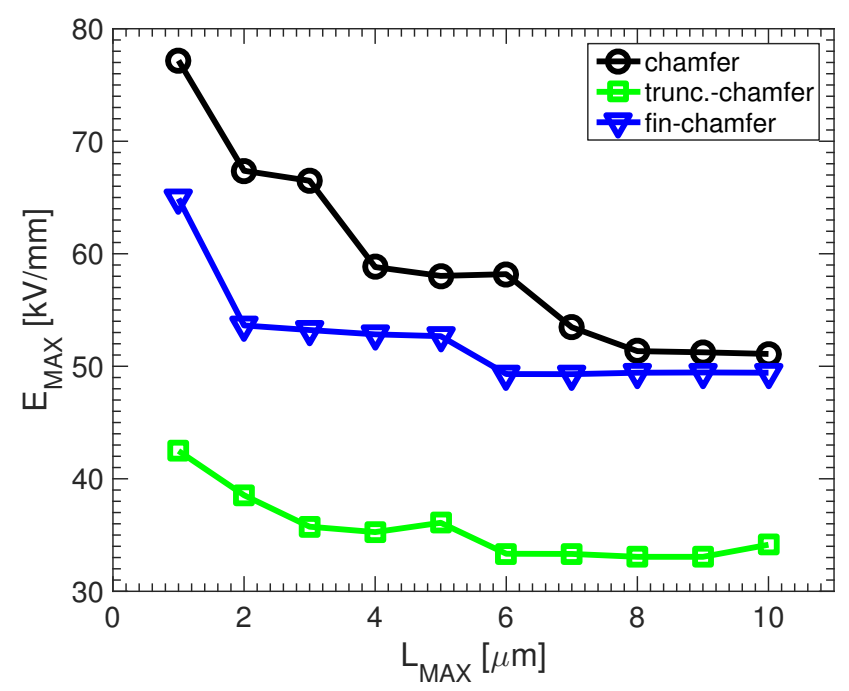

Figure 10: $E_{\mathrm{MAX}}$ vs. $L_{\mathrm{MAX}}$ for the three singularity shapes under investigation. The sharpness of the singularity endtip and the mesh refinement strongly influence $E_{\mathrm{MAX}}$. Furthermore, even the $E_{\mathrm{MAX}}$ ratio among singularities depends on $L_{\mathrm{MAX}}$.

point singularity, both truncated-chamfer and fin-chamfer are actually dielectric-dielectric corners. To be reliable, the method should not provide drastically different results once the shape at the singularity end-tip undergoes slight geometric variations, even this corresponds to a modification of the singularity nature.

The calculated $E$ for the three different singularity shapes is shown in Figure 9 (a2), (b2), and (c2), for chamfer, truncated-chamfer, and fin-chamfer, respectively (with $\left.L_{\mathrm{MAX}}=1 \mu \mathrm{m}\right)$. It can be easily appreciated how $E_{\mathrm{MAX}}$ undergoes significant variations for the different singularity shapes. Qualitatively, $E_{\mathrm{MAX}}$ is higher for increased tip sharpness, or decreased "equivalent curvature", i.e. $E_{\mathrm{MAX}}$ is the highest for the chamfer, slightly lower for the finchamfer, and the lowest for the truncated-chamfer case ${ }^{1}$. If we perform the $E_{\mathrm{MAX}}$ analysis, we would be limited by focusing on the results shown in Figure 10, which predict significant difference on the calculated $E_{\mathrm{MAX}}$ varying the end-tip sharpness. Moreover, not only the absolute values, but even the ratio of the $E_{\mathrm{MAX}}$ of the three singularities depends on the mesh refinement: e.g. at $L_{\mathrm{MAX}}=2$ $\mu \mathrm{m}, E_{\mathrm{MAX}}$ of fin-chamfer is about the $57 \%$ of the chamfer $E_{\mathrm{MAX}}$; however, if $L_{\mathrm{MAX}} \geq 8 \mu \mathrm{m}$, chamfer and finchamfer features almost the same $E_{\mathrm{MAX}}$. These results confirm once against that the $E_{\mathrm{MAX}}$ metric is of very limited applicability, and that calculated $E_{\mathrm{MAX}}$ cannot be directly compared with the $E_{r}$.

Figure 11 shows the results of the application of the new approach. Correctly, the $E_{\mathrm{AVG}}$-metric predicts higher

\footnotetext{
${ }^{1}$ Note that the comparison is not affected by the fact that the singularity points are located at different $r$ : the increase in the radial coordinate of the singularity location for the truncated and the fin -chamfers is negligible, since it would yield a field reduction lower than two orders of magnitudes the minimum $E_{\mathrm{MAX}}$ difference among the singularity shapes.
}

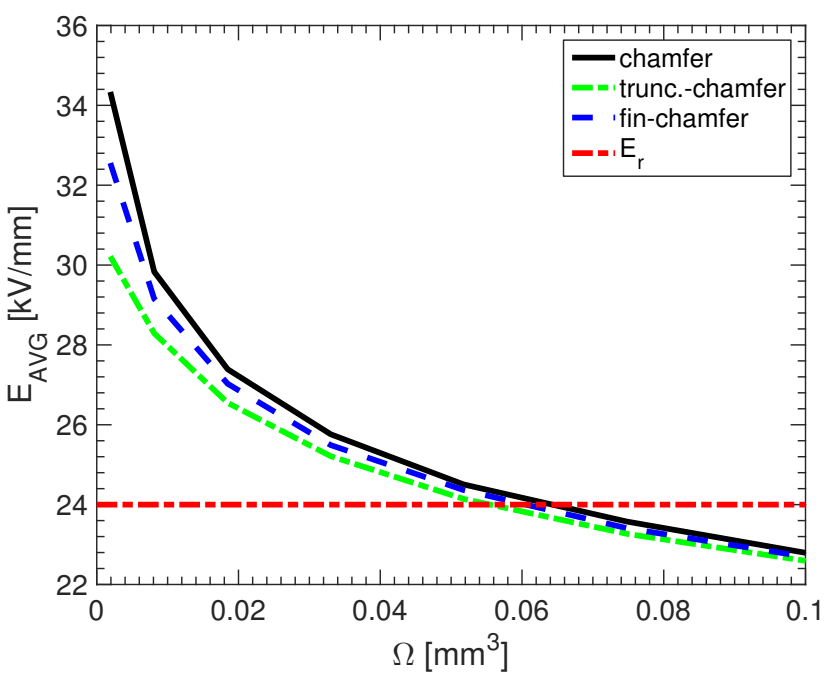

Figure 11: $E_{\mathrm{AVG}}$ as a function of $\Omega$ for the three investigated singularities. The variation of $E_{\mathrm{AVG}}$ with respect to the singularity end-tip shape is modest, though a monotonic increase of $E_{\mathrm{AVG}}$ for increased singularity sharpness can be appreciated. No dependence on $L_{\mathrm{MAX}}$ is found.

Table 1: Relative variations of $E_{\mathrm{MAX}}, E_{\mathrm{AVG}}$, and $\Omega_{\mathrm{C}}$ for the three singularities investigated. $E_{\mathrm{MAX}}$ compared at $L_{\mathrm{MAX}}=1 \mu \mathrm{m} ; E_{\mathrm{AVG}}$ compared at $\Omega=\Omega_{\mathrm{C}, \mathrm{AVG}}$, where $\Omega_{\mathrm{C}, \mathrm{AVG}} \sim 0.06 \mathrm{~mm}^{3}$ is the arithmetic average of the $\Omega_{\mathrm{C}}$ of the three investigated singularity shapes. The values are expressed in percentage using the chamfer as reference.

\begin{tabular}{l|ccc}
\hline Singularity & $E_{\mathrm{MAX}}[\%]$ & $E_{\mathrm{AVG}}[\%]$ & $\Omega_{\mathrm{C}}[\%]$ \\
\hline Chamfer & 100 & 100 & 100 \\
\hline Truncated-chamfer & 50 & 98.52 & 86 \\
\hline Fin-chamfer & 85 & 99.16 & 93 \\
\hline
\end{tabular}

field for chamfer, lower for fin-chamfer and the lowest for truncated-chamfer, accordingly to the tip sharpness. Then, more importantly, the $E_{\mathrm{AVG}}$ variations are much more limited and their relative magnitudes are pretty much constant on the whole curve. Obviously, the $E_{\mathrm{AVG}}$ gap among the three singularities tends to increase for lower $\Omega$ we are approaching the $E_{\mathrm{MAX}}$ analysis framework.

In Table 1, the calculated $E_{\mathrm{MAX}}, E_{\mathrm{AVG}}$, and critical volume $\Omega_{\mathrm{C}}$ are compared, in percentage, among the three singularity points. The $E_{\mathrm{MAX}}$ are calculated with $L_{\mathrm{MAX}}=1$ $\mu \mathrm{m}$, while the $E_{\mathrm{AVG}} \mathrm{S}$ are all taken at $\Omega=\Omega_{\mathrm{C}, \mathrm{AVG}}$, where $\Omega_{\mathrm{C}, \mathrm{AVG}} \sim 0.06 \mathrm{~mm}^{3}$ is the arithmetic average of the $\Omega_{\mathrm{C}}$ of the three singularities. The maximum $E_{\mathrm{AVG}}$ variation is lower than the $2 \%$, highlighting the very good robustness of the proposed methodology with respect to the high variability suffered by the $E_{\mathrm{MAX}}$ criterion, featuring a maximum variation of the $50 \%$.

\subsection{Sensitivity}

Another important assessment of the method reliability is the sensitivity test. Contrary to the robustness test, where 


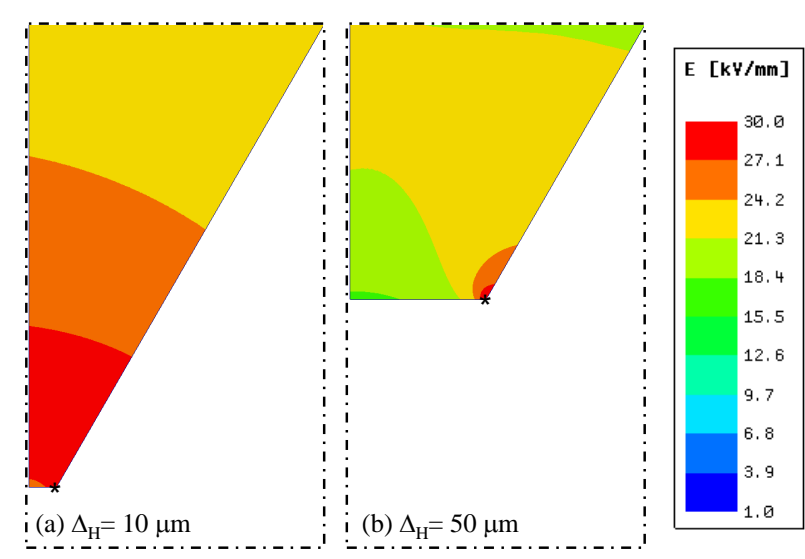

Figure 12: Electric field magnitude for truncated-chamfer singularities; (a) $\Delta_{\mathrm{H}}=10 \mu \mathrm{m}$, (b) $\Delta_{\mathrm{H}}=50 \mu \mathrm{m}$. The singularity points, in correspondence to which the control volumes are centered, are indicated by the asterisks $(*)$. The colormap legend ranges from $1 \mathrm{kV} / \mathrm{mm}$ to $30 \mathrm{kV} / \mathrm{mm}$.

we take different singularity shapes but of substantially the same size, in the following we investigate a same singularity shape but with a significant difference in size, hence in sharpness. Therefore, let us consider the truncated-chamfer singularity, which truncation height parameter $\Delta_{\mathrm{H}}$ is defined with respect to the original chamfer shape having $\Delta_{\mathrm{H}}$ $=0$, see Figure $9(\mathrm{~b} 1)$.

The $E$ of two truncated-chamfer singularities having the one $\Delta_{\mathrm{H}}=10 \mu \mathrm{m}$ (i.e. the same truncated-chamfer profile investigated so far) and the other a 5 times bigger $\Delta_{\mathrm{H}}=50$ $\mu \mathrm{m}$ is displayed in Figure 12. As expected, since to bigger $\Delta_{\mathrm{H}}$ corresponds bigger "equivalent curvature", i.e. lower sharpness, the electrical stress in the $\Delta_{\mathrm{H}}=50 \mu \mathrm{m}$ case is significantly reduced. The sharpness difference is correctly predicted by the averaging method, as shown in Figure 13 , where $E_{\mathrm{AVG}}$ is displayed against $\Omega$. Clearly, the $10 \mu \mathrm{m}$ curve is much more critical for the electrical stress standpoint, featuring a $\Omega_{\mathrm{C}}$ about 5 times higher than the one extracted on the $50 \mu \mathrm{m}$ curve. Indeed, the same numerical factor by which $\Delta_{\mathrm{H}}$ is increased is reflected in the $\Omega_{\mathrm{C}}$ decrease. To a lower $\Omega_{\mathrm{C}}$ corresponds a lower volume subjected to possible dielectric breakdown, hence a reduction of the probability of occurring in a device failure, for a given device operational time. This results make us confident of the sensitivity of the averaging field approach to correctly capture significant difference in the singularity sharpness shape.

\section{Discussion}

To summarize, the proposed methodology to analyze the results of electromagnetic FEM simulations at field singularities provides mesh independence, robustness and sensitivity.

However, in real applications, the electric stress analyst must cope with conceptual drawings of complex industry

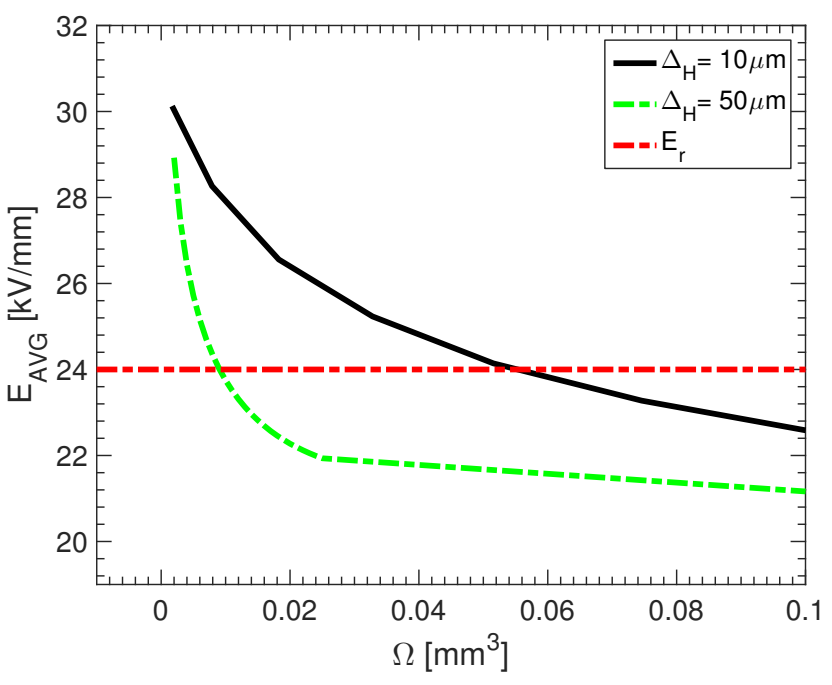

Figure 13: $E_{\mathrm{AVG}}$ vs. $\Omega$ of two truncated-chamfer singularities having $\Delta_{\mathrm{H}}=10 \mu \mathrm{m}$ and $\Delta_{\mathrm{H}}=50 \mu \mathrm{m}$. The respective critical volumes differ from about a factor of 5 , being the sharper singularity, i.e. $\Delta_{\mathrm{H}}=10 \mu \mathrm{m}$, the one featuring the higher $\Omega_{\mathrm{C}}$, hence the more critical. No dependence on $L_{\mathrm{MAX}}$ is found.

parts that are usually plenty of singularities, which shape details might not have been investigated in-depth. Therefore, for convenient application of the method proposed in this work, a preliminary understanding of the critical parts of the design is mandatory in order to better focus the analysis effort. To this aim, failure analysis data or critical information on the process conditions are obviously of paramount importance.

In addition, the analysis of the electric field can only partially address the complexity of the dielectric breakdown physics. To thoroughly evaluate the probability of partial or complete discharge in a device, the electromagnetic model should be coupled to the physical model for dielectric breakdown, such as impact ionization models, space charge models, chemical reactions at the electrodes, etc. [9]. On the other hand, especially when novel materials are employed, most of the critical physical parameters necessary to completely describe the breakdown physics are typically unknown and the simulation outcome is hardly predictive, despite the modeling effort put in place to include the breakdown model is remarkably higher compared to one needed for the pure electromagnetic analysis. For these reason, the relatively simple electromagnetic modeling is always the most convenient baseline for electrical reliability study, allowing the fast screening of many electrical stress grading options, being thus useful especially in the initial stage of a design process.

\section{Conclusions}

We presented a novel approach to analyze the results of FEM simulations in correspondence of field singularities. Our methodology provides the following advantages com- 
pared to the standard practice: (i) it makes the analysis independent of the mesh refinement at the model singularities, thus allowing direct comparison with respect to the material dielectric strength; (ii) it provides excellent robustness of the analysis outcome against unknown geometric details of the singularity shape; (iii) it ensures sensitivity with respect to significant variations of the singularity end-tip sharpness, for a given shape; (iv) it estimates the volume interested by the partial discharge, thus providing a simple mean for a first-level assessment of the device durability.

\section{References}

[1] R. E. Collin, Fields Theory of Guided Waves, $2^{\text {nd }} e d$. Oxford University Press, 1991.

[2] R. A. Hurd, The Edge Condition in Electromagnetics, IEEE Transactions on Antennas and Propagation, Vol. 24, pp. 70-73 (1976).

[3] F. Olyslager, The behavior of electromagnetic fields at edges in bi-isotropic and bi-anisotropic materials, IEEE Transactions on Antennas and Propagation, Vol. 42, pp. 1392-1397 (1994).

[4] L. Donzel and J. Schuderer, Nonlinear Resistive Electric Field Control for Power Electronic Modules, IEEE Transactions on Dielectrics and Electrical Insulation, Vol. 19, No. 3, pp. 955-959 (2012).

[5] M. Defourny, Multiple domain point for Laplace equation in the boundary element method, Engineering Analysis with Boundary Elements, Vol. 6, No. 3, pp. 136-139, (1989).

[6] Y. Oh, K. Song, H Choe, and S. Hahn, Advanced Finite-Element Method Employing a Singularity Function for Triple Junction Problems, IEEE Transactions on Magnetics, Vol. 45, n. 3, pp. 13361339, (2009).

[7] L. Krahenbuhl, F. Buret, R. Perrussel, D. Voyer, P. Dular, V. Peron, C. Poignard, Numerical treatment of rounded and sharp corners in the modeling of 2D electrostatic fields,Journal of Microwaves, Optoelectronics and Electromagnetic Applications, 10 (1), pp.6681 (2011).

[8] C.F. Bayer, E. Baer, U. Waltrich, D. Malipaard, and A. Schletz, Simulation of the Electric Field Strength in the Vicinity of Metallization Edges on Dielectric Substrates, IEEE Transactions on Dielectrics and Electrical Insulation, Vol. 22, No. 1, pp. 257-265 (2015).

[9] E. Kuffel, W.S. Zaengl, J. Kuffel, High Voltage Engineering: Fundamentals, $2^{\text {nd }} e d$. Newnes., 2000. 